\title{
Enhancement of Image Resolution: A Survey
}

\author{
Dimple Mittal \\ M.E Scholar \\ Thapar University \\ Patiala
}

\author{
Husanbir Singh Pannu \\ Lecturer \\ Thapar University \\ Patiala
}

\begin{abstract}
The High Resolution images have great importance in various fields, such as astronomy, medical imaging, agricluture, video surveillance, etc. These High Resolution images are useful to get the required details which are important for analysis in many applications. This paper investigates mainly on various modern existing methods of super resolution that and putting it all together for a literature survey. Scope of this study mainly focuses on the different available techniques of image processing to get high resolution images for extracting the meticulous details.
\end{abstract}

\section{Keywords}

SSIM, LISTA, HR images

\section{INTRODUCTION}

These days there is a tremendous increase in use of digital image processing and has brought up the demand of high resolution images for proper analysis in different domains. These domains are medical imaging, astronomy, video surveillance, agriculture and many more. The image's quality is measured on the basis of the pixel value that is resolution and it is considered as an important parameter.

As in medical field HR images are used to proper analyzing any body part or to detect any disease. In the field of astronomy it becomes easy for scientists to study any planet or any universe activities. Therefore, image resolution enhancement is a crucial area for the researchers. HR images are not always accessible. Due to shortcomings of the sensor and optics building methods, images with HR are not always achievable and are expensive.

Super resolution is an emerging technique to solve this difficulty; it uses image processing algorithms such as nearest neighbor, sparse coding and many more. Super-resolution (SR) is a process to estimate a HR image from a single noisy LR image. It means an image has high pixel density, and hence a HR image can depict other supplementary details that may be critical in applications e.g. A HR image could localize a tumor more accurately.

In the current era the resolution of an image is dependent on image acquisition devices. However, if device generates the image having high pixel value, so it means the cost of the device also get increases and hence it may not be an economical solution. Therefore, the proposed ideas emphasizes on other solutions rather than hardware updating solutions. As hardware updations are more costly and complex. Super Resolution algorithms are best solution for enhancing the resolution of the image.

\section{LITERATURE REVIEW}

To study more about the super-resolution techniques, following literature survey has done and discussed in the coming sections. The high resolution image gives more details about the original scene but it is not an effortless task to achieve an image of appropriate resolution due to the imaging environment and factors such as noise and blur that limits the quality of image. The only solution to this problem is SR methods that can be used for processing the image.

Due to the advancement in the technologies such as 3-D imaging, the demand for HR depth images is increasing. All the traditional methods of depth super resolution reconstructs HR images by retrieving details of the image either internally from HR image or externally from the database of HR images. Therefore, H. Zheng et al. presented a new method for depth super resolution. This new technique exploits both the internal and external HR information to obtain images having high pixel value. This new joint regularization method formed with different constraints, allows solving of HR image and sparse code simultaneously [4].

In [25], a new method is proposed for improving the resolution of a grayscale image. First the LR image is interpolated using Lancoz interpolation. Then extracting the features from interpolated image. Features are extracted using Lancoz and Gradient filter. The PCA is used get the most efficient information of feature extraction. Dictionary is trained using k-SVD algorithm. Than sparse representation algorithm is performed and finally image is reconstructed.

The k-nearest neighbor method given by [12] is very simple. It find out the closest pixel value to the specify input pixel. After that it gives that value to the output pixel. This method does not find out new pixel values. It just copies the already present values as it does not change the values.

Trinh, Luong, Dibos et al. has jointly put forward a novel example-based method [16]. They proposed the method for denoising and to increase the resolution of medical images. In this method, resolution is improved by using database of HR and LR patches. Their main objective is estimation of HR images from a single noisy low resolution image. The estimation is done on the basis of nonnegative sparse representation of the input patch. Both Denoising and SR are performed on each patch. The nonnegative sparse linear representation can be found for the input taken as a nonnegative quadratic equation.

Russo proposed an approach to enhance contrast of image [3]. In this method, there is fuzzy model for sharpening of image. This method has better performance than the earlier methods presented in the field of image enhancement affected by Gaussian noise. Fuzzy set parameters does not involve complicated calculation.

Hong Chang, Dit-Yan Yeung et.al discussed a novel method to solve single-image SR problems [5]. Low resolution image is inputted and corresponding to this equivalent high resolution image is reconstructed. This reconstruction is done 
using set of training examples. The proposed technique is motivated by numerous learning methods, especially from locally linear embedding. The patches in low and high resolution images create different forms with same local geometry in two different feature spaces. Apart from training image pairs, method also impose smoothness and local compatibility constraints to solve the problem.

In Today's era, many applications require the images with high contrast and sharpness. Guang Deng presented a generalized unsharp masking algorithm [6]. The designed algorithm confronts the three issues: enhancing the contrast and intensity by method of isolated treatment of the components and the residue of model, how to reduce halo effect with the method of an edge-preserving filter, and to solve the problem of out-of-range using log-ratio and tangent operations. The properties of log-ratio approach eliminate rescaling process. In the proposed algorithm, there is availability to adjust the parameters which control the contrast and intensity to get the required results.

Another new scaling algorithm of SR is introduced [7] in which the obtained HR image depicts more accurate details of edges. With this algorithm the image is enlarged to any size without uneven surface or blurring factors. Four steps are performed in the scaling process: (i) edge orientation is calculated, (ii) average is computed for the edge orientation, (iii) detection of edge patterns, and (iv) interpolation of the pixel values. These all are pipelined to obtain efficient implementation. The performance is evaluated on the basis of Structural Similarity Index (SSIM).

Yang et al. proposed sparse representation method for improving the resolution of image and removing the noise [11]. Two dictionaries are prepared from examples. It involves sparse association between the HR patch and LR patch. The basic idea behind this method is to show an input vector as a weighted linear combination of small number of basis atoms. It is performed in two parts: (i) training is done for two dictionaries. One dictionary for LR patches and one for HR patches by using example images. Then, (ii) reconstruction of HR image is done. The Sparse Coding SR (ScSR) method chooses patch from the dictionary that best represents LR patch. This algorithm depends on sparse association between image patches and experimental result show great outcomes in robustness to image corruption. Its performance is much better than all other SRs method like Interpolation and k-Nearest Neighbor method.

A technique to obtain unknown data points within the range of discrete points is called interpolation. Hassan Aftab described a new technique for super resolution [8]. Aerial images are effectively demonstrated by using this technique. It is a fast hybrid method to switch the covariance based interpolation (that is interpolation of edges) with curvature based interpolation (interpolation based on second order directional derivatives of image intensity) techniques. This method is applied to the areas on the grounds of edge and smooth. The presented method shows better visual quality and less processing time.

[9] uncovered a new technique to resample the discrete data known as Cubic Convolution interpolation. The new technique function converges to the interpolation function as sampling increment. By observing the conditions of boundary and constraints of the interpolation kernel, the accuracy of method lies in between linear interpolation and cubic spline The author derived a one-dimensional interpolation function.

$$
g(x)=\sum_{k} c_{k} u\left(\frac{x-x_{k}}{h}\right)
$$

where $\mathrm{h}$ represents the sampling increment, the $\mathrm{x}_{\mathrm{k}}$ 's represent the interpolation nodes, $\mathrm{u}$ is kernel, and $\mathrm{g}$ is the function defined for interpolation. $\mathrm{C}_{\mathrm{k}}$ 's defined are parameters that depend on the sampled data.

The Magnetic Resonance Imaging (MRI) systems are affected from poor out-of-plane resolution. Post-acquisition, SR filtering is feasible and less expensive, off-line image processing approach. Souza et al. introduced a new SR framework in [10] which improves resolution of tissues and contrast of acquired 3D MRI. The framework models the acquired information on the basis of slice thickness and space in between slices. Available SR techniques did consider the type of acquisition information i.e. sampling the data. This framework shows better results than existing method in the field of synthetic data and clinical knee MRI data.

Liu et.al discussed sparse coding model which is used to improve the resolution of an image using key concepts of deep learning [1]. On the basis of Learned Iterative Shrinkage and Thresholding Algorithm (LISTA) network, a Neutral Network is implemented for every step of sparse coding processing. All the components of sparse coding are trained through back propagation. Back propagation trains the network on the basis of gradient descent method.

William C. Karl et al. proposed a technique for medical images [13]. The technique is used to lessen the blooming effect of the bright object that is caused due to low-dose imaging system. In this framework an image is specified as composition of two components. The first one is defined by first function and the second one by second function. This method generates the medical image by minimizing the first and second function using a smoothing process.

Cao Qi invented a technique image super resolution algorithm [14]. The algorithm is based on sparse representation and UV Chroma Processing. On the basis of color shades two digital images are combined using special effects is called Chroma Processing. Each patch is expressed as sparse representation. This representation is used to obtain high resolution image by learning dictionary. UV Chroma processing is used to further improve the effects of resolution by exploiting luminance information. The quality of image is measured in RMSE (Root-Mean-Square Error).

\section{PERFORMANCE PARAMETERS}

The performance of any high resolution image is measured by two important parameters, Peak Signal-To-Noise Ratio (PSNR) and Mean Squared Error (MSE). MSE is the measure of the difference between desired response and actual output. It can be obtained as:

$$
M S E=\frac{\sum_{x, y}\left(f_{p}(x, y)-f_{o}(x, y)\right)^{2}}{M \times N}
$$

where $\mathrm{M} \times \mathrm{N}$ is the size of an image, $f_{p}(x, y)$ is the processed image and $f_{o}(x, y)$ is the original image.

PSNR is implemented to obtain quantitative results for comparison. Larger the PSNR value, the better will be image quality. PSNR can be obtained by: 


$$
P S N R=10 \log _{10}\left(\frac{M A X I}{M S E}\right)
$$

where $R$ is the maximum fluctuation an can image have.

In Fig(1) it can be observed that a low resolution image is intensified using the SR technique to get a high resolution image.

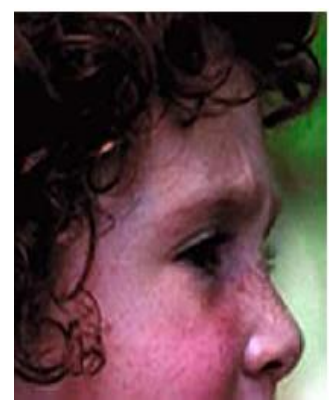

Figure: 1(a)

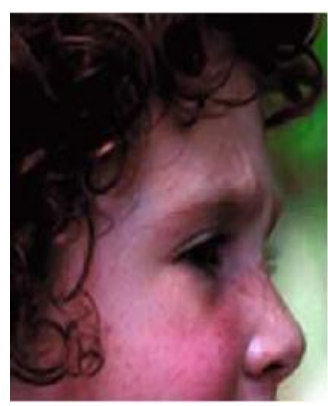

Figure: 1(b)

\section{CONCLUSION}

From the study of these algorithms it has been observed that the intensity of image can be intensified. Today many applications use the digital images to analyze the image in depth to take crucial decisions. As it is observed from different algorithms that digital images also plays vital role in medical field. A high resolution image makes the analyzing process easy. Any disease can be identified and body part is studied by zooming the specific part of the image. So, intensification of the images makes it easy for the observer to analyze the image easily by zooming specific areas

\section{REFERENCES}

[1] Wang, Z. 2015. Ding Liu, Jianchao Yang, Wei han. Deep Networks for Image Super-Resolution with Sparse Prior. IEEE (Dec 2015), 370-378.

[2] Dinh-Hoan, Luong, M., Dibos, F., Rocchisani, J.M. 2014. Novel Example-Based Method for SuperResolution and Denoising of Medical Images. IEEE Trans. Signal Processing, April 2014.

[3] F.Russo. 2002. An image enhancement technique combining sharpening and noise reduction. IEEE Transactions on Instrumentation and Measurement. Aug 2002, 824-828.
[4] Zheng, H., Bouzerdoum, A., Phung, S.L. 2015 Depth image super-resolution using internal and external information. IEEE International Conference on Acoustics, Speech and Signal Processing, Aug 2015.

[5] Chang, H., Yeung, D.-Y., Xiong, Y. 2004 Superresolution through neighbor embedding. CVPR.

[6] Deng, G. 2011. A Generalized Unsharp Masking Algorithm. IEEE Transactions on Image Processing, 1249-1261.

[7] Okuhata, H., Omaki, R.Y. 2013. Implementation of super-resolution scalar for Full HD and 4K video. IEEE Third International Conference on Consumer Electronics, Sept 2013.

[8] Aftab, H., Mansoor, A.B., Asim, M. 2008. A new single image interpolation technique for super resolution. IEEE International, , Dec 2008, 592-596.

[9] Keys, R. 1981. Cubic convolution interpolation for digital image processing, in Acoustics, Speech and Signal Processing. IEEE Transactions., Dec 1981, 11531160 .

[10] Senn, A.R. 2008. Model-based super-resolution for MRI. IEEE, Aug 2008, , 430-434.

[11] Yang, J., Wright, Huang, J., Yi Ma, T.S. 2010. Image Super-Resolution Via Sparse Representation in Image Processing. IEEE Transactions, Nov 2010, 2861-2873.

[12] Dong, W., Fu, F. 2016 Hyperspectral Image SuperResolution via Non-Negative Structured Sparse Representation. IEEE Transactions On Image Processing, May 2016.

[13] Qi, C., Zhu G., Ji X., Zhao, L. Image super resolution reconstruction algorithm based on sparse representation and the UV chroma processing.

[14] Progress in Informatics and Computing (PIC). 2014 International Conference on, IEEE, May 2014, 368-372.

[15] Ramos, V.A., and Ponomaryov, V. 2016. Sparse Representation to Solve the Problem of Image SuperResolution, IEEE Conference Proceeding Conielecomp, Feb , 2016. 